\title{
Uso de bioativador, bioestimulante e complexo de nutrientes em sementes de soja ${ }^{1}$
}

\author{
José Adolfo Binsfeld ${ }^{2}$, Ana Paula Piccinin Barbieri², \\ Caroline Huth $^{2}$, Ingrid Cervo Cabrera ${ }^{2}$, Liliane Marcia Mertz Henning ${ }^{3}$
}

\begin{abstract}
Use of bioactivator, biostimulant and complex of nutrients in soybean seeds

New discoveries have stimulated the use of different substances with physiologic effects, in order to develop agricultural crops. Thus, this study aimed at evaluating seeds treated with biostimulant, bioactivator and nutrients, in the initial development of soybean seeds. Two lots of seeds (high and low vigor, BMX Potência RR cultivar) were used. The products tested were an insecticide with bioactivator effect, a plant growth regulator with biostimulant effect, a complex of nutrients and a control. Under laboratory conditions, the parameters water content, germination, first germination counting, accelerated aging, cold test, length and dry matter weight of seedlings were evaluated. Under greenhouse conditions, evaluations included emergence, emergence speed index, length and dry matter weight of seedlings. The efficiency of the products tested was affected by the seed physiologic quality, with a more pronounced effect for the products in high vigor lots. In general, the treatment with best results for initial performance was the complex of nutrients, followed by the plant growth regulator with biostimulant effect. The bioactivator had negative effect on seeds germination and seedling development.
\end{abstract}

KEY-WORDS: Glycine max L.; seed vigor; seed treatment.

\section{INTRODUÇÃO}

A soja é a principal oleaginosa cultivada no mundo, sendo a cultura agrícola brasileira que apresentou maior crescimento, nas últimas décadas, ocupando, aproximadamente, $50 \%$ da área agrícola plantada (Conab 2013), o que contribui, significativamente, para a economia do País. Dentre os fatores que contribuem para assegurar o bom desempenho da cultura, está a utilização de sementes de alta qualidade, capazes de proporcionar adequado estabelecimento da cultura no campo.

\section{RESUMO}

Novas descobertas têm estimulado a utilização de diferentes substâncias com efeitos fisiológicos, com vistas a maior expressão no desenvolvimento de culturas agrícolas. Assim, objetivou-se avaliar o efeito do tratamento de sementes com bioestimulante, bioativador e nutrientes, no desenvolvimento inicial de sementes de soja. Foram utilizados dois lotes de sementes (alto e baixo vigor) da cultivar BMX Potência RR. Os produtos testados foram inseticida com efeito bioativador, regulador de crescimento vegetal com efeito bioestimulante, complexo de nutrientes e testemunha. Em laboratório, foram avaliados os parâmetros teor de água, germinação, primeira contagem de germinação, envelhecimento acelerado, teste de frio, comprimento e massa de matéria seca de plântulas. Em casade-vegetação, as avaliações incluíram a emergência, índice de velocidade de emergência, comprimento e massa de matéria seca de plântulas. A eficiência dos produtos testados sofreu influência da qualidade fisiológica da semente, sendo observado efeito mais pronunciado dos produtos em lotes de alto vigor. De forma geral, o tratamento que apresentou melhor resultado no desempenho inicial foi o complexo de nutrientes, seguido pelo regulador de crescimento vegetal com efeito bioestimulante. O bioativador teve efeito negativo sobre a germinação das sementes e sobre o desenvolvimento das plântulas.

PALAVRAS-CHAVE: Glycine max L.; vigor de sementes; tratamento de sementes.

A qualidade de um lote de sementes é determinada pela sua condição genética, sanitária, física e fisiológica. A caracterização da qualidade fisiológica das sementes é baseada, fundamentalmente, no teste de germinação, o qual já possui metodologia padronizada. Porém, por ser conduzido em condições ideais, o teste possui limitações, como a inabilidade para detectar diferenças de qualidade entre lotes com alta germinação (Hampton \& Tekrony 1995). Neste sentido, para a avaliação da qualidade fisiológica de sementes, de forma ampla e segura, é necessária a

1. Trabalho recebido em abr./2013 e aceito para publicação em mar./2014 ( $n^{\circ}$ registro: PAT 23474).

2. Universidade Federal de Santa Maria (UFSM), Faculdade de Agronomia, Departamento de Fitotecnia, Santa Maria, RS, Brasil. E-mails: jose_a_binsfeld@hotmail.com, apaulabarbieri@yahoo.com.br, carol.huth@hotmail.com, ingrid_cervo@hotmail.com.

3. Empresa Brasileira de Pesquisa Agropecuária, Embrapa Soja, Londrina, PR, Brasil. E-mail: liliane.henning@embrapa.br. 
complementação das informações do teste de germinação com testes de vigor, que permitem estimar o potencial de emergência de plântulas em campo, sob ampla faixa de condições de ambiente (Barros et al. 2002).

O tratamento de sementes de soja vem sendo vastamente adotado, pois, além de permitir a germinação de sementes infectadas, controla patógenos transmitidos pelas sementes e protege as sementes dos fungos do solo, possibilitando maior potencial para o desenvolvimento inicial da cultura e estabelecimento do estande inicial, a custos reduzidos (Henning 2005). Além de fungicidas, inseticidas e nematicidas, produtos que alteram o crescimento e desenvolvimento vegetal, como reguladores de crescimento vegetal e micronutrientes, podem ser utilizados no tratamento de sementes.

Alguns inseticidas podem conferir, além do efeito protetor, certos tipos de efeitos fisiológicos, auxiliando tanto no crescimento inicial quanto no desenvolvimento das plantas (Dan et al. 2012). Estes produtos são, comumente, chamados bioativadores, ou seja, são substâncias orgânicas complexas, capazes de modificar o crescimento das plantas (Costa 2010). Trabalhos relatam que o princípio ativo deste inseticida proporcionou maior vigor em plântulas de milho, ervilha, soja (Horrii et al. 2007), trigo (Macedo \& Castro 2011) e arroz (Grohs et al. 2012), além de proteger as plântulas de soja contra estresses abióticos (seca) (Cataneo et al. 2010), confirmando seu poder bioativador.

Os controladores hormonais, ou reguladores de crescimento vegetal, têm despertado atenção cada vez maior, no agronegócio, à medida em que as técnicas de cultivo evoluem, principalmente em culturas de grande importância econômica, como a soja. Dentre eles, pode-se citar os bioestimulantes, que são substâncias sintéticas, constituídas por misturas de um ou mais biorreguladores com outros compostos quimicamente diferentes, como os sais minerais, e que provocam alterações nos processos vitais e estruturais da planta (Castro \& Vieira 2001).

Além da utilização desses produtos, outra tecnologia que vem sendo adotada no tratamento de sementes é a suplementação destas com nutrientes. Estudos realizados em diversas regiões do Brasil sugerem a ocorrência de deficiência ou toxidez aguda de vários elementos no solo, sendo o Mo, Co, Zn, $\mathrm{Cu}, \mathrm{Mn}$ e B os de maior frequência de deficiência, principalmente em solos do Cerrado (Sfredo \&
Oliveira 2010). Dentre os micronutrientes mais utilizados no tratamento de sementes estão o Co, Mo e $\mathrm{Zn}$, sendo os dois primeiros mais utilizados na soja, tendo ação direta no desenvolvimento e função dos nódulos de fixação de nitrogênio e na redução do nitrato e fixação do nitrogênio, respectivamente (Taiz \& Zeiger 2009).

Esses produtos podem atuar em diferentes fases do desenvolvimento das plantas, e o conhecimento de seus efeitos fisiológicos é fundamental, pois pode se tornar uma estratégia, a fim de garantir adequado estabelecimento da cultura.

Diante do exposto, este estudo objetivou avaliar a eficiência do tratamento de sementes de soja com bioestimulante, bioativador e complexo de nutrientes, no crescimento inicial de plântulas.

\section{MATERIAL E MÉTODOS}

O experimento foi conduzido no Laboratório Didático e de Pesquisas em Sementes e em casa-de-vegetação do Departamento de Fitotecnia da Universidade Federal de Santa Maria (UFSM), sendo constituído por duas etapas, uma em laboratório e outra em vasos e canteiros, respectivamente, de outubro a dezembro de 2012.

Foram utilizadas sementes de soja da cultivar BMX Potência RR, que apresenta ciclo semiprecoce (140 dias, aproximadamente), pertencente ao grupo de maturação 6,7, com hábito de crescimento indeterminado. As sementes foram provenientes da safra $2011 / 2012$, originadas de dois lotes com diferentes níveis de vigor (alto e baixo). Os tratamentos de sementes foram os seguintes: 1) testemunha sem tratamento; 2) inseticida com efeito bioativador; 3) complexo de nutrientes; 4) regulador de crescimento vegetal com efeito bioestimulante.

Os produtos e respectivas doses utilizadas foram: bioativador Tiametoxam $70 \mathrm{~g}$ i.a. $100 \mathrm{~kg}^{-1}$ de semente (Cruiser 350 FS $^{\circledR}$ ), na dose de $200 \mathrm{~mL}$ $100 \mathrm{~kg}^{-1}$ de sementes; complexo de nutrientes $\mathrm{N}(3 \%)$, $\mathrm{P}(5 \%), \mathrm{K}(1 \%), \mathrm{Ca}(1 \%), \mathrm{Mg}(0,5 \%), \mathrm{B}(0,5 \%), \mathrm{Co}$ $(0,2 \%), \mathrm{Cu}(0,5 \%), \mathrm{Mn}(2 \%), \mathrm{Mo}(10 \%)$, Ni $(0,1 \%)$ e Zn (1\%), na dose de $200 \mathrm{~mL} 100 \mathrm{~kg}^{-1}$ de sementes (Dimicron TMSp); e bioestimulate $0,5 \mathrm{~g} \mathrm{~L}^{-1} \mathrm{de}$ ácido indol butírico (auxina), 0,9 $\mathrm{g} \mathrm{L}^{-1}$ de cinetina (citocinina) e $0,5 \mathrm{~g} \mathrm{~L}^{-1}$ de ácido giberélico (giberelina), na dose de $600 \mathrm{~mL} 100 \mathrm{~kg}^{-1}$ de semente (Stimulate ${ }^{\circledR}$ ). A testemunha foi tratada com água destilada e o tratamento das sementes realizado em sacos plásticos, 
com capacidade para 3,0 L, utilizando-se $500 \mathrm{~g}$ de sementes por saco.

Após o tratamento das sementes, foram realizados os respectivos testes e determinações, para avaliar o efeito dos produtos na germinação, vigor e desempenho inicial das plântulas de soja.

As avaliações em laboratório foram as seguintes:

a) Teor de água: determinado pelo método da estufa a $105^{\circ} \mathrm{C} \pm 2^{\circ} \mathrm{C}$, por 24 horas, com circulação forçada de ar (Brasil 2009);

b) Germinação: foram utilizadas quatro repetições de 100 sementes, semeadas em rolos de papel germitest umedecidos 2,5 vezes o peso do papel seco e mantidos em germinador regulado a $25^{\circ} \mathrm{C}$. As avaliações foram realizadas aos cinco e oito dias após o início do teste (Brasil 2009), sendo os resultados expressos em percentagem de plântulas normais;

c) Comprimento de plântulas: foram utilizadas quatro repetições de 20 sementes. As sementes foram colocadas para germinar à temperatura de $25^{\circ} \mathrm{C}$, utilizando-se, como substrato, rolos de papel umedecidos 2,5 vezes o peso do papel seco. As avaliações foram realizadas aos sete dias após a semeadura (Nakagawa 1999), medindo-se o comprimento (parte aérea, raiz e total) em 15 plântulas normais, por repetição, retiradas aleatoriamente. Os resultados foram expressos em comprimento médio da parte área, raiz e total, por plântula, em centímetros;

d) Massa de matéria seca de plântulas: as 15 plântulas avaliadas no comprimento de plântulas foram separadas, com o auxílio de um bisturi, a fim de remover apenas os cotilédones. Em seguida, as plântulas inteiras (sem os cotilédones) foram acondicionadas em sacos de papel e colocadas para secar em estufa com circulação de ar, a $80^{\circ} \mathrm{C}$, durante 24 horas. Após este período, as amostras foram retiradas da estufa, colocadas em dessecador e, em seguida, pesadas, determinando-se a massa de matéria seca total das plântulas, sendo os resultados expressos em mg plântula-1 (Nakagawa 1999);

e) Teste de envelhecimento acelerado: realizado, inicialmente, em caixa tipo gerbox, onde foram adicionados $40 \mathrm{~mL}$ de água e colocada uma tela de arame galvanizado, sobre a qual foram distribuídas as sementes. As caixas foram fechadas e levadas a uma estufa incubadora, por um período de 48 horas, à temperatura de $41^{\circ} \mathrm{C}$ (Marcos Filho 1999). Logo após, as sementes foram submetidas ao teste de germinação, e a avaliação ocorreu aos cinco dias após a instalação do teste, com quatro amostras de 100 sementes, seguindo-se a mesma metodologia descrita anteriormente;

f) Teste de frio: realizado com quatro repetições com 50 sementes, as quais foram semeadas em papel germitest e colocadas em geladeira, a $10^{\circ} \mathrm{C}$, por cinco dias. Após este período, foram acondicionadas, por mais quatro dias, em $\mathrm{BOD}$, a $25^{\circ} \mathrm{C}$, procedendo-se, em seguida, à contagem de plântulas normais (Barros et al. 1999).

As avaliações em casa-de-vegetação foram as seguintes:

a) Emergência e índice de velocidade de emergência: realizadas em canteiros, em delineamento de blocos casualizados (DBC), com cinco blocos, contendo, cada um, 50 sementes de cada tratamento. As anotações de emergência foram realizadas diariamente, sendo que, no $15^{\circ}$ dia, o número de plântulas emergidas estabilizou-se, finalizando, assim, as avaliações. A emergência final foi obtida pela soma do total das plântulas emergidas em cada bloco de cada tratamento. O índice de velocidade de emergência foi obtido por meio de fórmula proposta por Maguire (1962);

b) Desenvolvimento de plântulas: foi realizado um experimento em vasos (volume de $400 \mathrm{ml}$ ), em $\mathrm{DBC}$, com quatro repetições, perfazendo 16 unidades experimentais. O solo (tipo de solo) foi homogeneizado, peneirado e misturado, na proporção de volume $2: 1$, com vermiculita (duas partes de solo e uma de vermiculita), antes de ser acondicionado nos vasos. Foi realizada a semeadura de três sementes por vaso, à profundidade de $3,0 \mathrm{~cm}$. As avaliações foram realizadas aos 8 e 16 dias após a semeadura, sendo que, em uma plântula por recipiente, para cada período, foram determinados os parâmetros de comprimento e massa de matéria seca, conforme descrito anteriormente, nas avaliações em laboratório.

Para a análise estatística, as variáveis foram submetidas à análise de variância e as médias comparadas pelo teste de Scott-Knott, a 5\%, utilizando-se o software Sisvar (Ferreira 2008).

\section{RESULTADOS E DISCUSSÃO}

Os resultados da análise de variância, para os testes realizados em laboratório, revelaram efeitos significativos $(p>0,05)$ entre os tratamentos, nas variáveis avaliadas, exceto para o envelhecimento acelerado (EA), comprimento de parte aérea (CA) 
e massa de matéria seca (MS), evidenciando que os produtos utilizados no tratamento das sementes de soja apresentaram resultados diferentes, quando comparados entre si.

Quanto ao efeito de lotes, observou-se significância em todas as variáveis, exceto para o teste de frio. Foi observado que o coeficiente de variação $(\mathrm{CV})$, nestas variáveis, apresentou valores abaixo de $10 \%$, o que demonstra a baixa variabilidade dos dados e maior confiança nos resultados. A interação entre tratamentos e lotes foi significativa para a maioria das variáveis analisadas, exceto para envelhecimento acelerado e teste de frio, demonstrando que o efeito do produto depende, também, da qualidade fisiológica das sementes.

Os resultados da análise de variância, para os testes realizados em casa-de-vegetação, revelaram efeitos significativos $(p>0,05)$ entre os tratamentos, para as variáveis emergência e índice de velocidade de emergência, demonstrando que os produtos apresentaram efeitos diferentes entre si, quando se utilizou solo, como substrato. Quanto aos comprimentos e massa de matéria seca de plântulas, avaliados aos 8 e 16 dias, houve diferença para o comprimento radicular, total e massa de matéria seca, aos 8 dias, e comprimento total e massa de matéria seca, aos 16 dias.

Considerando-se o efeito de lote, houve significância para emergência e índice de velocidade de emergência, e para as variáveis de comprimento de raiz, parte aérea e total, avaliadas aos 8 dias, e massa de matéria seca, aos 16 dias. Quanto à interação entre os tratamentos e os lotes, houve efeito significativo para comprimento radicular e total, aos 8 dias, e mas- sa de matéria seca, aos 16 dias, demonstrando que, para estas variáveis, a eficiência dos produtos testados variou de acordo com a qualidade das sementes.

Segundo os resultados, foi verificado que, para a variável germinação, houve desempenho superior com o uso dos produtos com efeito bioativador e complexo de nutrientes, no lote de menor vigor (Tabela 1). Em trabalhos utilizando tiametoxam, Castro et al. (2007) afirmam que as sementes de soja tratadas com este ingrediente ativo resultaram em germinação acelerada das sementes, por estimularem a atividade de enzimas, tendo, além disto, alcançado emergência uniforme, estande adequado e melhor arranque inicial. $\mathrm{O}$ efeito do produto está baseado no fato de que, dentro da planta, ele é transportado e é responsável por ativar várias reações fisiológicas, sendo, uma delas, a expressão de proteínas da membrana (Almeida et al. 2011).

Por outro lado, Dan et al. (2012), verificando o efeito do uso de tiametoxam em soja (dose de $0,15 \mathrm{~kg}$ de i.a. $/ 100 \mathrm{~kg}$ de sementes), não observaram diferenças significativas, quanto à germinação, índice de velocidade de germinação, comprimento radicular e de plântulas, envelhecimento acelerado, massa de matéria seca e altura, aos 30 dias após a semeadura, com relação à testemunha. Tavares et al. (2007), avaliando o efeito fisiológico da aplicação de diferentes doses de tiametoxam $(0 \mathrm{ml}, 50 \mathrm{ml}, 100 \mathrm{ml}, 200 \mathrm{ml} \mathrm{e}$ $300 \mathrm{ml}$ do produto comercial por $100 \mathrm{~kg}$ de sementes), em soja, também não observaram diferença significativa entre os tratamentos, para germinação, comprimento de hipocótilo e radícula.

Com relação ao efeito positivo do tratamento das sementes com o complexo de nutrientes sobre

Tabela 1. Germinação (G), primeira contagem (PC), envelhecimento acelerado (EA), emergência (E), índice de velocidade de emergência (IVE), comprimento radicular (CRA), comprimento da parte aérea (CPA), comprimento total (CT), massa de matéria seca de plântulas (MS) e teste de frio (F) de lotes de sementes de soja tratadas com bioativador, bioestimulante e complexo de nutrientes (Santa Maria, RS, 2012).

\begin{tabular}{|c|c|c|c|c|c|c|c|c|c|c|c|}
\hline \multirow{2}{*}{ Lote $^{1}$} & \multirow{2}{*}{ Tratamento } & $\mathrm{G}$ & $\mathrm{PC}$ & EA & $\mathrm{E}$ & \multirow{2}{*}{ IVE } & CRA & CPA & $\mathrm{CT}$ & \multirow{2}{*}{$\begin{array}{c}\mathrm{MS} \\
\mathrm{mg}\end{array}$} & \multirow{2}{*}{$\begin{array}{c}\mathrm{F} \\
\%\end{array}$} \\
\hline & & \multicolumn{4}{|c|}{$\longrightarrow \%$} & & & $-\mathrm{cm}-$ & & & \\
\hline \multirow{4}{*}{1} & Testemunha & $56 \mathrm{c}$ & $56 \mathrm{c}$ & $75 \mathrm{a}$ & $83,0 \mathrm{a}$ & $9,054 \mathrm{a}$ & $16,27 \mathrm{a}$ & $10,33 \mathrm{a}$ & $26,60 \mathrm{a}$ & $0,474 \mathrm{a}$ & $87 \mathrm{a}$ \\
\hline & Bioativador & $89 a$ & $86 a$ & $65 \mathrm{a}$ & $69,0 \mathrm{~b}$ & $7,562 \mathrm{~b}$ & $13,38 \mathrm{~b}$ & $8,46 \mathrm{~b}$ & $21,84 \mathrm{c}$ & $0,487 \mathrm{a}$ & $83 \mathrm{a}$ \\
\hline & C. nutrientes & $86 \mathrm{a}$ & $86 a$ & $70 \mathrm{a}$ & $77,0 \mathrm{a}$ & $8,672 \mathrm{a}$ & $14,26 \mathrm{~b}$ & $10,00 \mathrm{a}$ & $24,26 \mathrm{~b}$ & $0,490 \mathrm{a}$ & $83 \mathrm{a}$ \\
\hline & Bioestimulante & $68 \mathrm{~b}$ & $65 \mathrm{~b}$ & $81 \mathrm{a}$ & $82,0 \mathrm{a}$ & $9,068 \mathrm{a}$ & $16,42 \mathrm{a}$ & $10,42 \mathrm{a}$ & $26,84 \mathrm{a}$ & $0,457 \mathrm{a}$ & $77 \mathrm{a}$ \\
\hline \multirow{4}{*}{2} & Testemunha & $93 \mathrm{a}$ & $90 \mathrm{a}$ & $80 \mathrm{a}$ & $77,2 \mathrm{a}$ & $8,550 \mathrm{a}$ & $16,58 \mathrm{~b}$ & $10,61 \mathrm{a}$ & $27,19 \mathrm{a}$ & $0,510 \mathrm{~b}$ & $84 \mathrm{a}$ \\
\hline & Bioativador & $80 \mathrm{~b}$ & $78 \mathrm{~b}$ & $85 \mathrm{a}$ & $67,6 \mathrm{a}$ & $7,182 \mathrm{a}$ & $17,18 \mathrm{~b}$ & $11,07 \mathrm{a}$ & $28,25 \mathrm{a}$ & $0,518 \mathrm{~b}$ & $76 \mathrm{~b}$ \\
\hline & C. nutrientes & $84 \mathrm{~b}$ & $82 \mathrm{~b}$ & $83 \mathrm{a}$ & $71,2 \mathrm{a}$ & $7,656 \mathrm{a}$ & $15,22 \mathrm{c}$ & $10,07 \mathrm{a}$ & $25,29 \mathrm{~b}$ & $0,530 \mathrm{~b}$ & $83 \mathrm{a}$ \\
\hline & Bioestimulante & $85 \mathrm{~b}$ & $83 \mathrm{~b}$ & $83 a$ & $72,0 \mathrm{a}$ & $7,676 \mathrm{a}$ & $18,08 \mathrm{a}$ & $10,50 \mathrm{a}$ & $28,58 \mathrm{a}$ & $0,555 \mathrm{a}$ & $75 \mathrm{~b}$ \\
\hline
\end{tabular}

* Médias seguidas pelas mesmas letras, nas colunas, não diferem entre si, pelo teste de Scott-Knott ( $\mathrm{p}>0,05)$. ${ }^{1}$ Lotes: 1 - baixo vigor; 2 - alto vigor. 
a germinação, Bays et al. (2007), utilizando micronutrientes (Co, Mo e B), no tratamento de sementes de soja, juntamente com fungicida e polímero, não observaram aumento na germinação e qualidade fisiológica das sementes, isto porque o Mo, Co e B são nutrientes importantes à fixação biológica do nitrogênio na soja, em fases posteriores às avaliadas. Já Guerra et al. (2006) observaram que o Mo $\left(74,1 \mathrm{~g} \mathrm{ha}^{-1}\right)$ e o $\mathrm{Co}\left(1,0 \mathrm{~g} \mathrm{ha}^{-1}\right)$ incrementam a germinação de sementes de soja.

Nas avaliações de comprimento de raiz e massa de matéria seca de plântulas, no lote 2 , ocorreu desempenho superior das sementes tratadas com o bioestimulante (Tabela 1), o que pode ter influência da cinetina (citocinina), pela divisão celular, e do ácido 4-indol 3-ibutírico (auxina), pelo gravitropismo e alongamento celular que promove nas plantas (Taiz \& Zeiger 2009). Este resultado vai de encontro aos obtidos por Santos (2009), que observou que o bioestimulante aumenta a percentagem de germinação de sementes, proporcionando incremento significativo sobre a massa de matéria seca das raízes das plântulas de soja.

Para o lote de maior vigor, aos 8 dias, utilizando-se solo, como substrato, pôde-se observar maior comprimento de raiz, parte aérea e massa de matéria seca de plântulas, no tratamento com o complexo de nutrientes, em relação aos demais (Tabela 2). Este efeito pode estar atrelado à maior disponibilidade de nutrientes à semente, na fase inicial, favorecendo o seu crescimento e acúmulo de fitomassa. Pôde-se observar que este comportamento se estendeu até os 16 dias, para os tratamentos com o complexo de nutrientes e com bioestimulante, que apresentaram resultados superiores para comprimento e massa de matéria seca de plântulas (Tabela 2), confirmando os resultados encontrados em laboratório.

Esses resultados concordam com os encontrados por Silva et al. (2012), que, avaliando o comprimento radicular de cultivares de soja tratadas com o produto Dimicron TMSp, observaram maior comprimento radicular das plântulas, quando comparadas à testemunha. Beltrame (2009) também observou que a aplicação de fertilizantes (macro e micro), via tratamento de sementes, permitiu maior desenvolvimento inicial do sistema radicular, principalmente em condições de deficiência hídrica.

Quanto à utilização de tiametoxam como bioativador, não foram observados resultados significativos que proporcionassem alguma alteração positiva no comprimento radicular ou total de plântulas, comparativamente à testemunha e aos outros tratamentos (Tabela 2). Estes resultados estão de acordo com os encontrados por Tavares et al. (2007) e Castro et al. (2008), que não encontraram diferença no desenvolvimento do hipocótilo e radícula de plântulas de soja submetidas a cinco doses de tiametoxam, no tratamento de sementes.

Quanto à emergência e índice de velocidade de emergência, de modo geral, para o lote 2 (de maior vigor), pôde-se notar que o tratamento com tiametoxam apresentou efeito negativo sobre estas variáveis (Tabela 1). Este resultado discorda de Dan et al. (2012), para os quais o tratamento de sementes com o inseticida tiametoxam não interferiu, negativamente, no desenvolvimento inicial das plântulas, na dose de $0,15 \mathrm{~kg}$ do i.a./ $100 \mathrm{~kg}$ de sementes.

No laboratório, em condições controladas, pôde-se verificar desempenho superior do regulador

Tabela 2. Comprimento radicular (CRA), comprimento de parte aérea (CPA), comprimento total (CT) e massa de matéria seca (MS), avaliados aos 8 e 16 dias após a semeadura de dois lotes de sementes de soja tratadas com bioativador, bioestimulante e complexo de nutrientes (Santa Maria, RS, 2012).

\begin{tabular}{|c|c|c|c|c|c|c|c|c|c|}
\hline \multirow{3}{*}{ Lote $^{1}$} & \multirow{3}{*}{ Tratamento } & \multicolumn{4}{|c|}{ Avaliação aos 8 dias após a semeadura } & \multicolumn{4}{|c|}{ Avaliação aos 16 dias após a semeadura } \\
\hline & & CRA & $\mathrm{CPA}$ & $\mathrm{CT}$ & MS & CRA16 & CPA16 & CT16 & MS16 \\
\hline & & & $-\mathrm{cm}$ & & $\mathrm{mg}$ & & $-\mathrm{cm}$ & & $\mathrm{mg}$ \\
\hline \multirow{4}{*}{1} & Testemunha & $8,25 \mathrm{a}$ & $4,95 \mathrm{a}$ & $13,20 \mathrm{a}$ & $0,0814 \mathrm{a}$ & $10,82 \mathrm{a}$ & $9,72 \mathrm{~b}$ & $20,54 \mathrm{a}$ & $0,2680 \mathrm{a}$ \\
\hline & Bioativador & $9,51 \mathrm{a}$ & $4,31 \mathrm{a}$ & $13,82 \mathrm{a}$ & $0,0826 \mathrm{a}$ & $10,59 \mathrm{a}$ & $10,19 \mathrm{a}$ & $20,78 \mathrm{a}$ & $0,3006 \mathrm{a}$ \\
\hline & C. nutrientes & $9,55 \mathrm{a}$ & $4,99 \mathrm{a}$ & $14,54 \mathrm{a}$ & $0,0916 \mathrm{a}$ & $11,53 \mathrm{a}$ & $10,51 \mathrm{a}$ & $22,04 \mathrm{a}$ & $0,3096 \mathrm{a}$ \\
\hline & Bioestimulante & $9,00 \mathrm{a}$ & $4,75 \mathrm{a}$ & $13,75 \mathrm{a}$ & $0,0788 \mathrm{a}$ & $10,89 \mathrm{a}$ & $9,34 \mathrm{~b}$ & $20,23 \mathrm{a}$ & $0,2602 \mathrm{a}$ \\
\hline \multirow{4}{*}{2} & Testemunha & $5,78 \mathrm{c}$ & $3,88 \mathrm{a}$ & $9,66 \mathrm{c}$ & $0,0844 \mathrm{~b}$ & $9,52 \mathrm{~b}$ & $9,90 \mathrm{~b}$ & $19,42 \mathrm{~b}$ & $0,2938 \mathrm{~b}$ \\
\hline & Bioativador & $6,27 \mathrm{c}$ & $3,25 \mathrm{~b}$ & $9,52 \mathrm{c}$ & $0,0734 \mathrm{~b}$ & $9,78 \mathrm{~b}$ & $9,65 \mathrm{~b}$ & $19,43 \mathrm{~b}$ & $0,2924 \mathrm{~b}$ \\
\hline & C. nutrientes & $10,80 \mathrm{a}$ & $4,47 \mathrm{a}$ & $15,27 \mathrm{a}$ & $0,1052 \mathrm{a}$ & $11,71 \mathrm{a}$ & $10,68 \mathrm{a}$ & $22,39 \mathrm{a}$ & $0,3622 \mathrm{a}$ \\
\hline & Bioestimulante & $7,94 \mathrm{~b}$ & $4,06 \mathrm{a}$ & $12,00 \mathrm{~b}$ & $0,0882 \mathrm{~b}$ & $10,93 \mathrm{a}$ & $10,71 \mathrm{a}$ & $21,64 \mathrm{a}$ & $0,3430 \mathrm{a}$ \\
\hline
\end{tabular}

* Médias seguidas pelas mesmas letras, nas colunas, não diferem entre si, pelo teste de Scott-Knott ( $\mathrm{p}>0,05)$. ${ }^{1}$ Lotes: 1 - baixo vigor; 2 - alto vigor. 
de crescimento vegetal com efeito bioestimulante. Já em casa-de-vegetação, foi observado efeito superior do tratamento com o complexo de nutrientes, seguido do tratamento com o bioestimulante, principalmente no lote de alto vigor, evidenciando, assim, a importância da realização do mesmo experimento em duas condições de ambiente.

\section{CONCLUSÕES}

1. A eficiência dos produtos testados sofreu influência da qualidade fisiológica da semente, sendo observado efeito mais pronunciado dos produtos em lotes de alto vigor.

2. De forma geral, não se observou efeito dos produtos testados sobre a germinação, porém, o complexo de nutrientes, seguido do regulador de crescimento vegetal com efeito bioestimulante, influenciaram, positivamente, no desempenho inicial de plântulas.

3. O bioativador tiametoxam apresentou efeito negativo sobre a germinação de sementes e desenvolvimento de plântulas.

\section{REFERENCIAS}

ALMEIDA, A. S. et al. Bioativador no desempenho fisiológico de sementes de arroz. Revista Brasileira de Sementes, Londrina, v. 33, n. 3, p. 501-510, 2011.

BARROS, A. S. R. et al. Testes de frio. In: KRZYZANOWSKI, F. C.; VIEIRA, R. D.; FRANÇANETO, J. B. (Eds.). Vigor de sementes: conceitos e testes. Londrina: Abrates, 1999. cap. 5, p. 1-15.

BARROS, D. I. et al. Comparação entre testes de vigor para avaliação da qualidade fisiológica de sementes de tomate. Revista Brasileira de Sementes, Londrina, v. 24, n. 2, p. 12-16, 2002.

BAYS, R. et al. Recobrimento de sementes de soja com micronutrientes, fungicida e polímero. Revista Brasileira de Sementes, Londrina, v. 29, n. 2, p. 60-67, 2007.

BELTRAME, L. C. Eficiência do uso de fertilizantes, fungicidas e inoculante no tratamento de sementes de soja. 2009. 62 f. Dissertação (Mestrado em Fitotecnia) - Escola Superior de Agricultura Luiz de Queiroz, Universidade de São Paulo, Piracicaba, 2009.

BRASIL. Ministério da Agricultura e Reforma Agrária. Regras para análise de sementes. Brasília, DF: MAPA, 2009.

CASTRO, P. R. C. et al. Análise da atividade reguladora de crescimento vegetal de tiametoxam através de biotestes. Revista UEPG, Ponta Grossa, v. 13, n. 1, p. 25-29, 2007.
CASTRO, G. S. A. et al. Tratamento de sementes de soja com inseticidas e um bioestimulante. Pesquisa Agropecuária Brasileira, Brasília, DF, v. 43, n. 10, p. 13111318, 2008.

CASTRO, P. R. C.; VIEIRA, E. L. Aplicações de reguladores vegetais na agricultura tropical. Guaíba: Agropecuária, 2001.

CATANEO, A. C. et al. Improved germination of soybean seed treated with thiamethoxam under drought conditions. Seed Science and Technology, Madison, v. 38, n. 1, p. 248251, 2010.

COMPANHIA NACIONAL DE ABASTECIMENTO (Conab). Acompanhamento de safra brasileira: grãos. Brasília, DF: Conab, 2013.

COSTA, N. de L. Bioestimulante como fator de produtividade da cana-de-açúcar. 2010. Disponível em: <http://www.infoteca.cnptia.embrapa.br/handle/ doc/878849>. Acesso em: 03 dez. 2012.

DAN, L. G. M. et al. Tratamento de sementes com inseticida e a qualidade fisiológica de sementes de soja. Revista Caatinga, Mossoró, v. 25, n. 1, p. 45-51, 2012.

FERREIRA, D. C. Sisvar: um programa para análises e ensino de estatística. Revista Symposium, Lavras, v. 6, n. 1, p. 36-41, 2008.

GHROS, M. et al. Desempenho de cultivares de arroz com uso de reguladores de crescimento, em diferentes sistemas de cultivo. Pesquisa Agropecuária Brasileira, Brasília, DF, v. 47, n. 6, p. 776-783, 2012.

GUERRA, C. A. et al. Qualidade fisiológica de sementes de soja em função da adubação com fósforo, molibdênio e cobalto. Acta Scientarum: Agronomy, Maringá, v. 28, n. 1, p. 91-97, 2006.

HAMPTON, J. G.; TEKRONY, D. M. Controlled deterioration test. In: HAMPTON, J. G.; TEKRONY, D. M. (Eds.). Handbook of vigour test methods. Zurich: ISTA, 1995. p. 70-78.

HENNING, A. A. Patologia e tratamento de sementes: noções gerais. Londrina: Embrapa Soja, 2005. (Documentos, 264).

HORII, A.; MCCUE, P.; SHETTY, K. Enhancement of seed vigour following insecticide and phenolic elicitor treatment. Bioresource Technology, Philadelphia, v. 98, n. 3, p. 623-632, 2007.

MACEDO, W. R.; CASTRO, P. R. de C. Thiamethoxam: molecule moderator of growth, metabolism and production of spring wheat. Pesticide Biochemistry and Physiology, San Diego, v. 100, n. 3, p. 299-304, 2011.

MAGUIRE, J. D. Speed of germination-aid in selection and evaluation for seedling emergence and vigor. Crop Science, Madison, v. 2, n. 1, p. 176-177, 1962. 
MARCOS FILHO, J. Teste de envelhecimento acelerado. In: KRZYZANOWSKI, F. C.; VIEIRA, R. D.; FRANÇANETO, J. B. (Eds.). Vigor de sementes: conceitos e testes. Londrina: Abrates, 1999. cap. 3, p. 1-24.

NAKAGAWA, J. Testes de vigor baseados no desempenho das plântulas. In: KRZYZANOWSKI, F. C.; VIEIRA, R. D.; FRANÇA NETO, J. B. (Eds.). Vigor de sementes: conceitos e testes. Londrina: Abrates, 1999. cap. 2, p. 2-24.

SANTOS, C. R. S. Stimulate na germinação de sementes, vigor de plântulas e no crescimento inicial de soja. 2009. 44 f. Dissertação (Mestrado em Ciências Agrárias) Universidade Federal do Recôncavo da Bahia, Cruz das Almas, 2009.
SFREDO, G. J.; OLIVEIRA, M. C. N. de. Soja: molibdênio e cobalto. Londrina: Embrapa Soja, 2010.

SILVA, A. N. et al. Influência de macro e micronutrientes sobre o crescimento de raízes e hipocótilo em diferentes cultivares de soja. In: MOSTRA DE INICIAÇÃO CIENTÍFICA, 15., 2012, Cruz Alta. Anais... Cruz Alta: Unicruz, 2012. p. 1-4.

TAIZ, L.; ZEIGER, E. Fisiologia vegetal. 4. ed. Porto Alegre: Artmed, 2009.

TAVARES, S. et al. Avaliação dos efeitos fisiológicos de tiametoxam no tratamento de sementes de soja. Revista de Agricultura, Piracicaba, v. 82, n. 1, p. 47-54, 2007. 